\title{
Antimicrobial resistance - Judicious use is the key
}

\author{
John M Conly MD
}

A ntibiotics are used as agents to prevent and treat infections caused by pathogenic bacteria and other microbes. Their discovery ranks as one of the most important developments of modern medicine. Thousands of years ago, the Chinese were aware of the therapeutic potential of mouldy soybean curd applied to carbuncles and furuncles, and the ancient Greeks routinely used agents with anti-infective properties (such as myrrh and inorganic salts) in the treatment of infected wounds (1). The discovery of penicillin by Alexander Fleming in 1928, followed by the discovery and clinical use of sulphonamides in the 1930s, heralded the age of modern antimicrobial chemotherapy $(1,2)$. Penicillin use became widespread in the 1940s. The use of sulphonamides and cephalosporins became widespread in the 1950s and 1960s, respectively.

Two pioneers in the early antimicrobial chemotherapy era recognized that antimicrobial resistance was a significant concern. Fleming (2) reported that microbes were educated to resist penicillin, and that a host of penicillin-fast organisms were bred out which could be passed to other individuals, eventually leading to a pneumonia or septicemia for which penicillin would be ineffective. In 1942, Dubos noted that a vacuum was created when entire microbial populations were severely reduced, causing a profound impact on the natural equilibrium between host and microbes (3). The phenomenon of antimicrobial resistance is an aspect of microbial ecology that developed almost immediately after the first antibiotics were used. This phenomenon represents a means of survival presented to a threatened microbial population, and occurs through genetic mutation, expression of a latent resistance gene or the acquisition of genes with resistance determinants $(4,5)$. These three occurrences are not mutually exclusive and may coexist within a given bacterium. The widespread use of antibiotics provides selective pressure favouring the propagation of resistant organisms. These selective pressures create opportunities for resistant strains to emerge, multiply and be transmitted. Levy (6) reminded us of possible ecological imbalances that may be established, and suggested that the reversibility of the selection process is dependent on repopulation by the original susceptible bacteria.

Antibiotic resistance is acknowledged as one of the most serious threats to the treatment of infectious diseases on a global basis. This threat prompted the World Health Organization to issue a warning that antibiotic resistance threatens to rob the world of opportunities to treat or cure many infectious diseases (7). Antibiotic-resistant organisms erode our therapeutic inventory and cause significant increases in the cost and toxicity of newer drugs (8).
Methicillin-resistant Staphylococcus aureus (MRSA), vancomycin-resistant enterococci (VRE), multiply antibioticresistant Shigella and Salmonella species, resistant enteric gramnegative bacilli (Escherichia and Klebsiella-Enterobacter species) and penicillin-resistant Streptococcus pneumoniae (PRSP) are examples of microorganisms with increasing rates of resistance to commonly used antimicrobials. Traditionally, PRSP and multiply resistant Shigella and Salmonella species are more common in the community setting, while MRSA, VRE and resistant enteric Gram-negative bacilli are more common in health care facilities.

The isolation of penicillin-resistant $S$ aureus was reported shortly after penicillin became widely available. Initially a sporadic occurrence, this type of resistance (borne by a plasmid) spread rapidly through the 1950s and 1960s. By the 1970s, almost all hospital strains and a large number of community strains of $S$ aureus were penicillin-resistant, and Canada was no exception. The development of semisynthetic penicillinaseresistant agents, methicillin and isoxazolyl penicillins in 1960 was followed by the report of MRSA in the early 1960s (9). MRSA has since been reported from both acute care and longterm care facilities in Canada throughout the past two decades $(10,11)$. Recent data from the Canadian Nosocomial Infection Surveillance Program (CNISP) revealed that the proportion of $S$ aureus isolates (representing both colonization and infection) reported as being methicillin-resistant increased from $1.0 / 100$ isolates $(0.5 / 1000$ admissions $)$ in 1995 (12) to $10 / 100$ isolates (5.4/1000 admissions) in 2003 (Health Canada, unpublished data). The majority of MRSA reports (69\%) (including both colonization and infection) were from central Canada, with the remainder from western (20\%) and eastern $(11 \%)$ Canada. This pattern of increase in the past eight years is disconcerting, with the majority of the isolates coming from acute care health care facilities. VRE was first reported in Canada in 1993 (13) and it has been recognized in all of the provinces, predominantly as colonization being found in surveillance cultures. The VRE Passive Reporting Network, established within the CNISP, identified 1315 instances of VRE throughout Canada between 1994 and 1998, with less than 5\% representing infection (14). Since 1999 (the year a prospective surveillance system was established within the CNISP), the rate of VRE isolates has increased from $0.15 / 100$ to $0.26 / 100$ enterococcal isolates (15). This rate is still relatively low, especially when compared with rates from the United States, where prevalence of nosocomial VRE increased from $0.3 \%$ in 1989 to $23 \%$ in $1999(16,17)$. Despite the geographical proximity of Canada and the United States,

Departments of Pathology and Laboratory Medicine, Medicine, and Microbiology and Infectious Diseases, Centre for Antimicrobial Resistance, University of Calgary, Calgary, Alberta

Correspondence: Dr John M Conly, Foothills Medical Centre, 1403-29 Street Southwest, Calgary, Alberta T2N 2T9.

Telephone 403-944-8222, fax 403-944-1095, e-mail jconly@ualberta.ca 
VRE has not attained the same colonization rate and is rarely a cause of infection. A prospective laboratory-based study (18) conducted within CNISP between 1999 and 2000 revealed relatively low rates of extended spectrum beta-lactamase-producing E coli $(0.26 \%)$ and Klebsiella species $(0.81 \%)$ in Canadian hospitals. This rate compares favourably with prevalence rates of $23 \%$ to $45 \%$ reported in studies from European and Latin American countries (19).

The prevalence of $S$ pneumoniae with reduced susceptibility to penicillin varies markedly throughout the world, with up to $70 \%$ resistance in Korea and $40 \%$ resistance in the United States $(20,21)$. In Canada, the prevalence of clinical isolates with reduced susceptibility to penicillin (both intermediateand high-level resistance) increased from less than $2 \%$ in the late 1980 s to $12.4 \%$ in 2000 (22). The prevalence of PRSP in a surveillance system of clinically-significant laboratory pneumococcal isolates from respiratory tract specimens from sites in nine provinces (23) has varied from $16.1 \%$ (13.7\% high-level and 2.4\% intermediate-level resistance) in 1999 to 2000, to 24\% (10.2\% high-level and 13.8\% intermediate-level resistance) in 2001 to 2002. According to another surveillance system collecting all clinically significant pneumococcal isolates from multiple tertiary care and community laboratories, the prevalence of PRSP was $15.0 \%$ (6.5\% high-level resistance and $8.5 \%$ intermediate-level resistance) in 2002 (24).

A study by McIsaac et al (25) describing uropathogen resistance in the community setting provided valuable data for practicing physicians. They assessed urine culture reports from 446 adult women from a random sample of primary care physicians in a community setting across Canada. They reported 235 positive urine cultures and found that $38 \%$ had resistance to at least one antibiotic and $21.5 \%$ had resistance to two or more antibiotics. The rate of resistance to ampicillin and trimethoprim-sulfamethoxazole (two commonly prescribed agents) was $38 \%$ and $11 \%$, respectively. As expected, the vast majority of the isolates (83\%) were E coli. The study suggested that rates of resistance were higher in western Canada than in eastern Canada, even after stratified analysis with other factors associated with resistance, including age, history of previous urinary tract infections and the type of organism identified (with resistance to ampicillin being the primary difference). The reasons for this observation are unexplained, although possible reasons may relate to sampling differences (over $50 \%$ of the isolates were from a single province), differences in the rates of obtaining urine cultures or differences in antimicrobial usage. The authors acknowledged that further studies with larger sample sizes are needed to corroborate the reproducibility of this difference. Single-drug resistance was significantly more common in women aged 65 years and older, which was not an unexpected finding. The authors pointed out that the resistance rates for uropathogens collected from tertiary care centre outpatient settings in Canada (26) may have overestimated the resistance rates to uropathogens. This is an important observation that underscores the need to carefully define the patient population from which resistance data are collected because inappropriate interpretation of resistance data can have important implications for antimicrobial selection. On a more encouraging note, a study (26) reported a 30\% rate of ampicillin resistance to $E$ coli, which is lower than the rates reported in similar patient populations in the United States (40\%) and the United Kingdom (49\%). In addition, an 11\% rate of trimethoprim-sulfamethoxazole resistance suggests that current guidelines for empirical treatment of urinary tract infections in Canada for uncomplicated cystitis with this agent are appropriate.

Controlling antimicrobial resistance is a difficult task that requires a multifaceted approach (27), including reducing unnecessary drug prescribing in both human and animal populations, reducing transmission of resistant organisms through enhanced infection control and environmental hygienic practices and identifying trends in resistance through surveillance. The overuse of antibiotics is considered to be a major factor in promoting the emergence and dissemination of antibiotic resistance. Many factors lead to unnecessary antimicrobial prescribing practices, such as patient expectations and demands, the desire of the physician to give the best possible treatment regardless of costs or downstream effects, failure to consider alternative treatments, inappropriate use of diagnostic laboratory studies, adequacy of the physician's knowledge and management of patients with infectious diseases, medicolegal considerations and the belief that the newer and broader spectrum agents represent the most effective treatment. McIsaac and To (28) studied the prescribing habits of family physicians in Canada for lower respiratory tract infections and reported that reasons for unnecessary prescribing included uncertainty about the need for antibiotics (69.6\%), the possibility that illness would intensify $(36.1 \%)$ and pressure from patients to prescribe $(32.8 \%)$.

Although some evidence suggests that the overall prescribing rates are decreasing in Canada (29), physicians and the general public must continue to be educated about the judicious use of antibiotics. Judicious prescribing habits and appropriate use continue to be key goals in controlling antimicrobial resistance and achieving an ecological balance between susceptible and resistant microbes in humans. Judicious prescribing habits and appropriate use require input from all individuals involved in the drug prescribing process, including physicians, dentists, nurse practitioners, veterinarians, pharmacists, farmers and the public. A multifaceted and multidisciplinary approach, coupled with enabling and reinforcing strategies that encourage change, offers an opportunity for success in controlling antimicrobial resistance (30).

\section{REFERENCES}

1. Weinstein L. General considerations. In: Goodman LS, Gilman A, eds. The Pharmacological Basis of Therapeutics. New York: Macmillan, 1970:1154.

2. Fleming A. Nobel Lecture, December 11, 1945. Nobel e-Museum. <http:/www.nobel.se/medicine/laureates/1945/fleming-lecture.html> (Version current at September 14, 2004).

3. Moberg CL. René Dubos: A harbinger of microbial resistance to antibiotics. Microb Drug Resist 1996;2:287-97

4. Gold HS, Moellering RC. Antimicrobial-drug resistance. N Engl J Med 1996;335:1445-53.

5. Davies J. Inactivation of antibiotics and the dissemination of resistance genes. Science 1994;264:375-82.

6. Levy S. Antibiotic resistance: An ecological imbalance. Ciba Found Symp 1997;207:1-14.

7. World Health Organization. Overcoming antimicrobial resistance. $<$ http://www.who.int/infectious-disease-report/2000/index.html> (Version current at September 14, 2004).

8. Casellas JM, Blanco MG, Pinto ME. The sleeping giant. Antimicrobial resistance. Infect Dis Clin North Am 1994;8:29-44.

9. Panlilio AL, Culver DG, Gaynes RP, et al. Methicillin-resistant Staphylococcus aureus in US hospitals, 1975 to 1991. Infect Control Hosp Epidemiol 1992;13:582-6. 
10. Simor AE, Augustin A, Ng J, Betschel S, McArthur M. Control of MRSA in a long-term care facility. Infect Control Hosp Epidemiol 1994;15:69-70.

11. Vortel JJ, Bell A, Farley JD, Shaw C, Guite A, Harper B. Methicillin-resistant Staphylococcus aureus (MRSA) in a British Columbia hospital - 1990. Can Dis Wkly Rep 1991;17:71-2.

12. Simor AE, Ofner-Agostini M, Bryce E, et al. The evolution of methicillin-resistant Staphylococcus aureus in Canadian hospitals: 5 years of national surveillance. CMAJ 2001;165:21-6.

13. Kibsey PC, Willey B, Low DE, Cain D, Boychuk LR, Heule M. Vancomycin-resistant Enterococcus faecium: First Canadian isolate. 61st Conjoint Meeting of Infectious Diseases. Vancouver, November 8 to 10, 1993:K5. (Abst)

14. Conly JM, Ofner ME, Paton S, et al. The emerging epidemiology of vancomycin-resistant enterococci in Canada 1993-1998: Results from the Canadian Nosocomial Infection Surveillance Program (CNISP) Passive Reporting Network. Can J Infect Dis 2001;12:364-70.

15. Johnston L, Ofner-Agostini M, Gravel D, et al, for the Canadian Hospital Epidemiology Committee and the Canadian Nosocomial Infection Surveillance Program. Vancomycin-resistant enterococci in Canadian health care facilities: 5 years of prospective surveillance (1998-2003). Infectious Diseases Society of America, Boston, September 30 to October 3, 2004.

16. Centers for Disease Control and Prevention. Nosocomial enterococci resistant to vancomycin - United States 1989-1993. MMWR Morb Mortal Wkly Rep 1993;42:597-9.

17. Martone WJ. Spread of vancomycin-resistant enterococci: Why did it happen in the United States? Infect Control Hosp Epidemiol 1998;19:539-45.

18. Mulvey MR, Bryce E, Boyd D, Ofner-Agostini M, et al. Ambler class A extended-spectrum beta-lactamase-producing Escherichia coli and Klebsiella spp in Canadian hospitals. Antimicrob Agents Chemother 2004:48:1204-14

19. Winokur PL, Canton R, Casellas JM, Legakis N. Variations in the prevalence of strains expressing an extended-spectrum betalactamase phenotype and characterization of isolates from Europe, the Americas, and the Western Pacific region. Clin Infect Dis 2001;32(Suppl 2):S94-103.

20. Wang E, Kellner JD, Arnold S. Antibiotic-resistant Streptococcus pneumoniae. Implications for medical practice. Can Fam Physician $1998 ; 44: 1881-8$.
21. Adam D. Global antibiotic resistance in Streptococcus pneumoniae. J Antimicrob Chemother 2002;50(Suppl 1):1-5.

22. Simor AE, Louie M, Low DE. Canadian national survey of prevalence of antimicrobial resistance among clinical isolates of Streptococcus pneumoniae. Canadian Bacterial Surveillance Network. Antimicrob Agents Chemother 1996;40:2190-3.

23. Zhanel GG, Palatnick L, Nichol KA, Bellyou T, Low DE, Hoban DJ. Antimicrobial resistance in respiratory tract Streptococcus pneumoniae isolates: Results of the Canadian Respiratory Organism Susceptibility Study, 1997 to 2002. Antimicrob Agents Chemother 2003;47:1867-74.

24. Powis J, McGeer A, Green K, et al. In vitro antimicrobial susceptibilities of Streptococcus pneumoniae clinical isolates obtained in Canada in 2002. Antimicrob Agents Chemother 2004;48:3305-11

25. McIsaac WJ, Mazzulli T, Moineddin R, Raboud J, Rodd S. Uropathogen antibiotic resistance in adult women presenting to family physicians with uncomplicated cystitis. Can J Infect Dis Med Microbiol 2004;15:266-70.

26. Zhanel GG, Karlowsky JA, Harding GKM, et al. A Canadian national surveillance study of urinary tract isolates from outpatients: Comparison of the activities of trimethoprimsulfamethoxazole, ampicillin, mecillinam, nitrofurantoin and ciprofloxacin. The Canadian Urinary Isolate Study Group. Antimicrob Agents Chemother 2000;44:1089-92.

27. Goldmann DA, Weinstein RA, Wenzel RP, et al. Strategies to prevent and control the emergence and spread of antimicrobialresistant microorganisms in hospitals. A challenge to hospital leadership. JAMA 1996;275:234-40.

28. McIsaac W, To T. Antibiotics for lower respiratory tract infections. Still too frequently prescribed? Can Fam Physician 2004;50:569-75.

29. Conly J. Antimicrobial resistance in Canada. CMAJ 2002;167:885-91.

30. Canadian Committee on Antibiotic Resistance. National Policy Conference on Antibiotic Resistance, October 5 to 7, 2002: Summary of proceedings. CCDR 2003;29:153-7. <http://www.hcsc.gc.ca/pphb-dgspsp/publicat/ccdr-rmtc/03pdf/cdr2918.pdf> (Version current at October 1, 2004). 


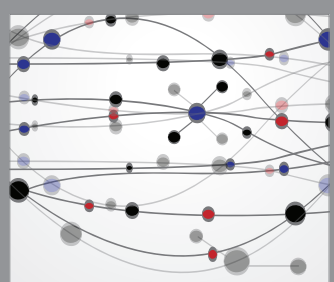

The Scientific World Journal
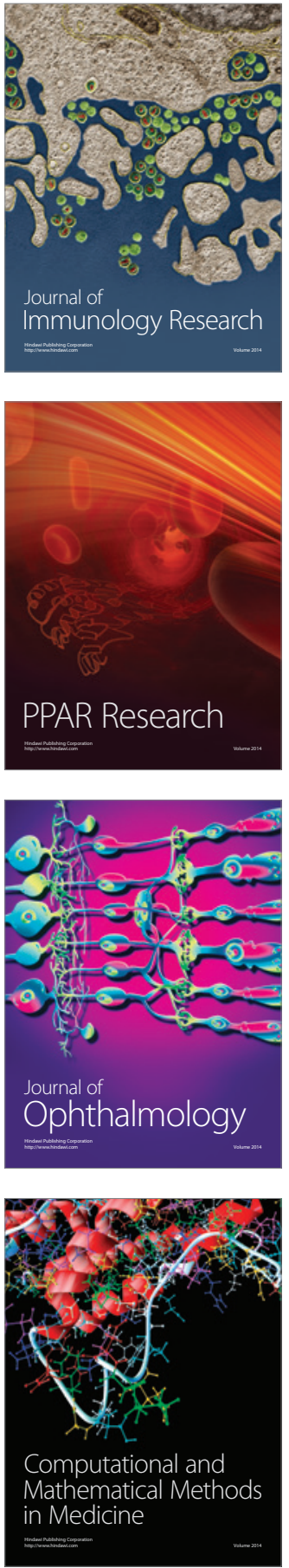

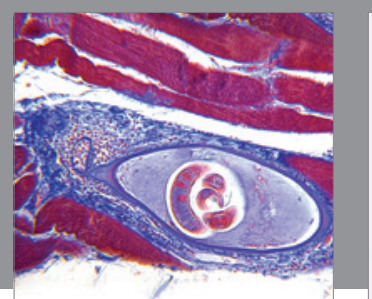

Gastroenterology Research and Practice

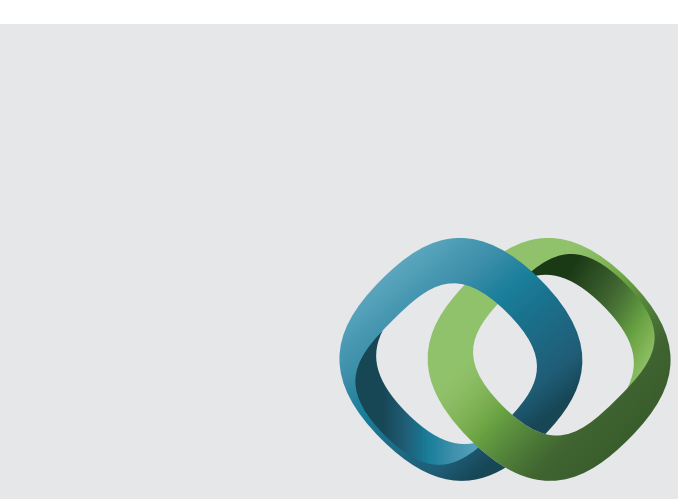

\section{Hindawi}

Submit your manuscripts at

http://www.hindawi.com
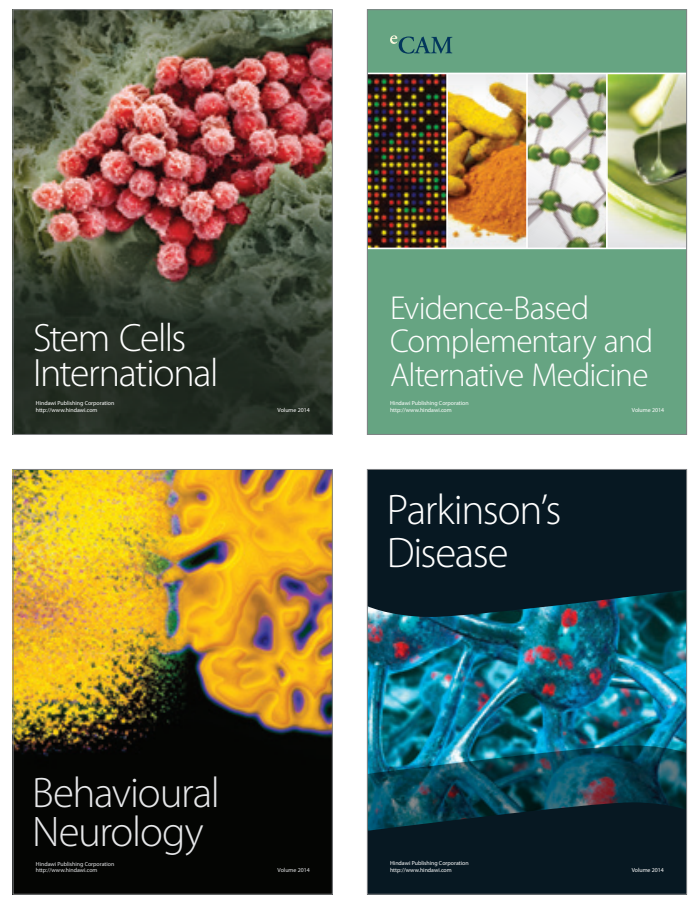
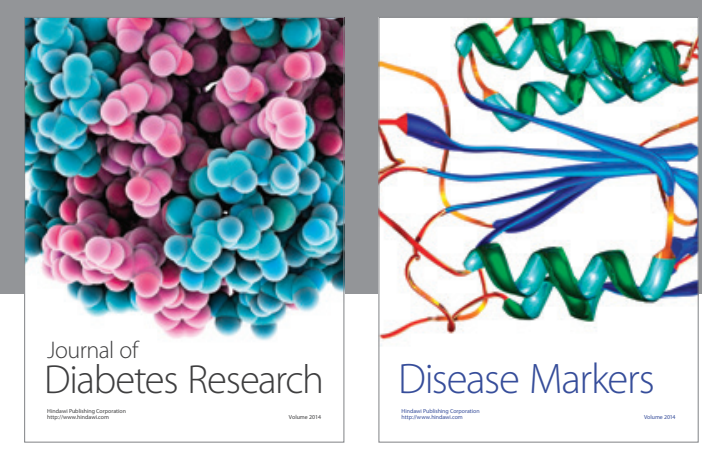

Disease Markers
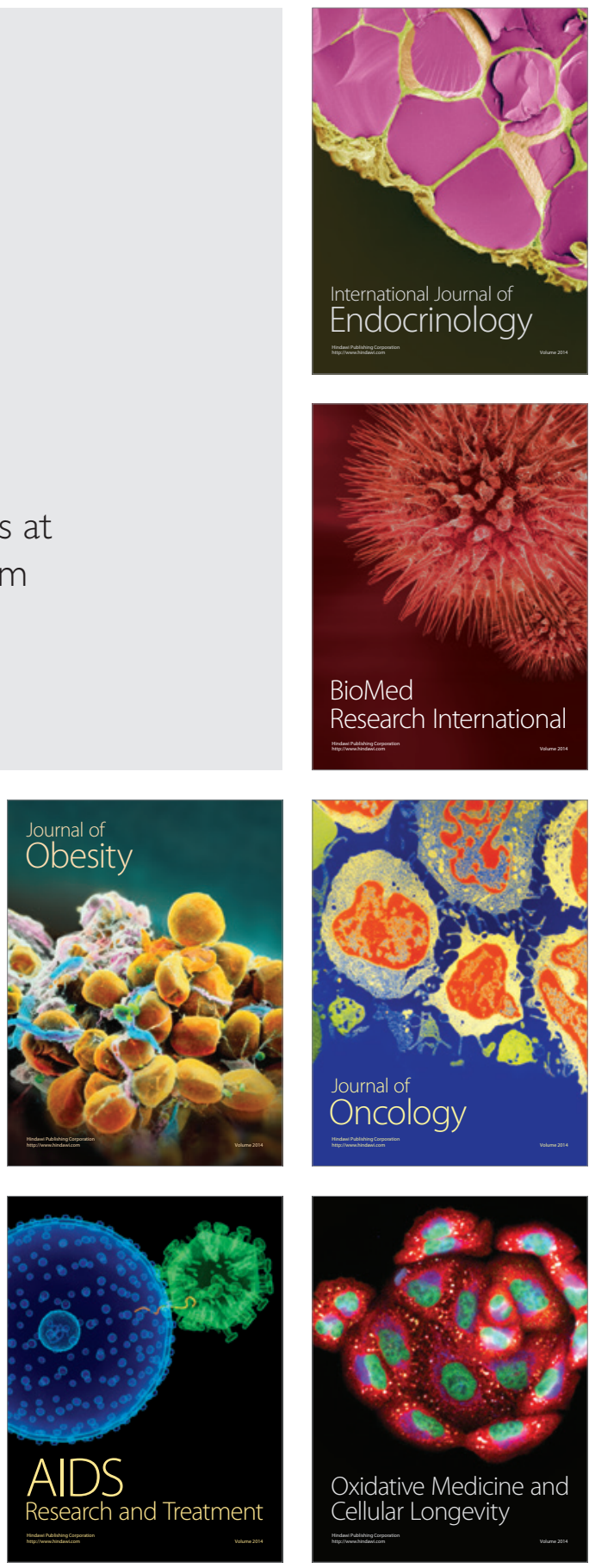